\title{
Health Information Exchange
}

National Cancer Institute

\section{Source}

National Cancer Institute. Health Information Exchange. NCI Thesaurus. Code C156650.

The process of sharing patient-level health information through electronic networks among disparate health information systems, primarily to permit immediate access at the point of care. 